\title{
Dengue en el embarazo: efectos en el feto y el recién nacido
}

\author{
Berta N. Restrepo ${ }^{1}$, Diana M. Isaza ${ }^{1,5}$, Clara L. Salazar ${ }^{1}$, José L. Ramírez ${ }^{2}$, \\ Gloria E. Upegui ${ }^{3}$, Marta Ospina ${ }^{4}$, Ruth Ramírez ${ }^{1}$ \\ 1 Instituto Colombiano de Medicina Tropical, Medellín, Colombia. \\ 2 Unidad de Genética Médica, Facultad de Medicina, Universidad de Antioquia, Medellín, Colombia. \\ ${ }^{3}$ ESE, Metrosalud, Medellín, Colombia. \\ ${ }^{4}$ Dirección Seccional de Salud de Antioquia, Medellín, Colombia. \\ ${ }^{5}$ Universidad de Antioquia, Medellín, Colombia. \\ El riesgo de infección por el virus del dengue durante el embarazo se está incrementando \\ ante mayores y más severas epidemias, y las consecuencias sobre el feto y el recién nacido \\ han sido poco estudiadas y, en otros casos, los resultados han sido contradictorios. Por esta \\ razón, se realizó en Medellín un estudio de cohorte retrospectiva, cuyo objetivo fue determinar \\ los efectos que produce el dengue durante el embarazo sobre el feto y el recién nacido. En \\ dicho estudio se evaluaron 22 recién nacidos hijos de mujeres que presentaron dengue durante \\ la epidemia de 1998 y se compararon con 24 recién nacidos, hijos de mujeres embarazadas \\ sin dengue. En la cohorte con dengue se encontraron 3 niños prematuros, 3 niños con \\ sufrimiento fetal y 4 niños con bajo peso al nacer. En la cohorte no expuesta no se encontraron \\ niños con estos problemas. El desarrollo psicomotor fue normal en ambos grupos. De las \\ observaciones anteriores, sólo fue estadísticamente significativa la frecuencia de niños con \\ bajo peso al nacer (prueba exacta de Fisher, $p=0,045$ ). Estos resultados preliminares muestran \\ que los recién nacidos de madres que sufrieron dengue durante la gestación tuvieron riesgo \\ de bajo peso al nacer y presentaron con mayor frecuencia prematurez y sufrimiento fetal, \\ aunque se requiere aumentar el tamaño de la muestra para confirmar estos resultados. Sin \\ embargo, es necesario estrechar la vigilancia a las madres embarazadas con dengue dados \\ los efectos nocivos sobre la evolución del recién nacido.
}

Palabras claves: dengue, embarazo, efectos sobre el feto y el recién nacido.

\section{Neonatal and postnatal effects of dengue infection during pregnancy}

The risk of dengue virus infection during pregnancy has increased due to the current rash of frequent and severe dengue epidemics. The effects of dengue virus in the fetus and newborn children have been studied only superficially and with contradictory results. Therefore, a retrospective cohort study was conducted in Medellin, Colombia, to describe the fetal and postnatal effects of dengue virus infection acquired during pregnancy. Twenty-two babies born from mothers who suffered dengue during the epidemics of 1998 were compared with babies from non-infected mothers. In the exposed cohort, three premature births occurred, three children suffered from fetal anomalies and four children were born with low weight. In the non-exposed children, none of these problems were found. Psychomotor development was normal in both groups. Only the low weight subgroup was statistically significant (Fisher test, $p=0.045)$. These results suggested that the children from women with dengue during pregnancy present low weight, greater frequency of premature birth and increased fetal distress. A larger sample is necessary to confirm these results.

Key words: dengue, pregnancy, affection in fetus and newborn. 
La infección por el virus del dengue se considera en la actualidad la arbovirosis humana más importante porque tiene inherentes una alta mortalidad y morbilidad. En Colombia, en el 2002, se registraron en todo el país 76.579 casos de dengue clásico, para una tasa de incidencia de 209,15 por 100.000 habitantes (1), la cual superó los años anteriores y 5.245 casos de dengue hemorrágico, para una tasa de incidencia de 14,33 por 100.000 habitantes (1). En Antioquia, el promedio anual de casos de dengue clásico en los últimos 5 años (1998-2002) fue de 3.357; 1998 fue el año de mayor frecuencia, con 9.746 casos (1) y la incidencia de dengue hemorrágico aumentó de 0,02 por 100.000 habitantes en 1989 a 4,27 por 100.000 en 1998 (2). Medellín, capital del departamento de Antioquia, tampoco ha estado exenta del problema y en 1998 se registraron 6.119 casos de dengue clásico y 119 de dengue hemorrágico, para una tasa de incidencia de dengue clásico de 310,5 por 100.000 habitantes.

El riesgo de infección en el embarazo se incrementa ante mayores y más severas epidemias de dengue y las consecuencias sobre el feto y el embarazo han sido poco estudiadas y, en otros casos, los resultados han sido contradictorios. Cantelar y Molina (3) informaron recién nacidos en buenas condiciones físicas al nacer, al igual que Chong y colaboradores (4), Figueiredo y colaboradores (5), Fernández y colaboradores (6) y Bunyavejchevin y colaboradores (7). Sin embargo, Sharma y Gulati (8) alertan sobre la posibilidad de malformaciones congénitas, y Carles y colaboradores (9-10) encuentran un serio riesgo de muerte fetal y prematurez. Por este motivo, se realizó un estudio retrospectivo para determinar los efectos del virus del dengue sobre el embarazo (11), y en el feto y el recién nacido.

\section{Materiales y métodos}

\section{Tipo de estudio}

El presente es un estudio de una cohorte retrospectiva.

$\overline{\text { Correspondencia: }}$

Berta Nelly Restrepo, Instituto Colombiano de Medicina Tropical, Carrera 43 A No. 52 Sur 99, Sabaneta, Antioquia Teléfonos: 3012135 y 30143 00; fax: 3014258 icmt@epm.net.co

Recibido: 16/06/03; aceptado: 10/10/03

\section{Tamaño de muestra}

El cálculo del tamaño de la muestra se hizo en Epilnfo, versión $6.04 \mathrm{c}$, con los siguientes parámetros estadísticos: nivel de confianza, $60 \%$; poder, $50 \%$; prevalencia de muerte fetal asociada con dengue, $13,6 \%$, y prevalencia de muerte fetal en población general, 1,9\%, según los datos del estudio de G. Carles y colaboradores en SaintLaurent du Maroni, Guyana Francesa (10). Resultó una muestra de 21 personas en cada grupo.

La cohorte expuesta (CE) correspondió a la totalidad de los recién nacidos hijos de mujeres gestantes que presentaron dengue durante la epidemia ocurrida en 1998, que fueron 22, ya que dos mujeres de este grupo sufrieron aborto en el primer trimestre de la gestación.

La cohorte no expuesta (CNE) correspondió a los recién nacidos hijos de mujeres que no presentaron síndrome febril durante el embarazo, procedentes de la misma zona geográfica de los casos y con un año de diferencia en edad, aproximadamente, para un total de 24 niños.

\section{Selección de la población de estudio}

Los recién nacidos de madres con dengue (CE) fueron captados de la base de datos del Laboratorio Departamental de Salud Pública de Antioquia, la cual incluía las muestras de todas las mujeres en edad reproductiva (13 a 49 años) referidas para estudio serológico de dengue y aislamiento viral procedentes de diferentes instituciones de salud de Medellín. Por contacto telefónico, se detectaron las mujeres que estaban en embarazo para incluirlas en el estudio con sus respectivos recién nacidos. Los recién nacidos de madres sin dengue (CNE) fueron seleccionados del listado de mujeres en control prenatal de la misma institución donde consultó la paciente con dengue. Se solicitó consentimiento informado por escrito a las participantes.

\section{Definición de términos}

Se consideró que una madre tenía enfermedad por dengue, si cumplía con los criterios de dengue contemplados en las definiciones operativas de los Protocolos de Vigilancia Epidemiológica de la Dirección Seccional de Salud de Antioquia $(11,12)$. Se consideró que una madre no tenía 
enfermedad por dengue, si durante el embarazo no había presentado síndrome febril, por interrogatorio y por historia clínica. Los posibles efectos del virus del dengue durante el embarazo sobre el feto y el recién nacido fueron los siguientes. Se consideró 'feto o recién nacido pretérmino' cuando el nacimiento había ocurrido antes de la semana 38 de gestación (13). Se diagnosticó 'sufrimiento fetal agudo' basándose en la interpretación de la frecuencia cardíaca fetal para evaluar la condición neonatal. 'Retardo en el crecimiento intrauterino', neonato que no alcanza el peso mínimo que corresponde con su edad gestacional (13). 'Niño de bajo peso al nacer' es todo recién nacido que pese $2.500 \mathrm{~g}$ o menos. 'Malformación congénita' es el defecto morfológico de un órgano, parte de un órgano o un área más grande del cuerpo que resulta de un proceso intrínseco anormal del desarrollo. El 'puntaje de Apgar' es un examen rápido que se realiza al primero y quinto minuto después del nacimiento para determinar la condición física; un puntaje menor de 8 indica que el niño necesita ayuda para estabilizarse.

\section{Diagnóstico de laboratorio}

La detección de anticuerpos IgM contra el virus del dengue a las madres se realizó por el método de microELISA disponible comercialmente en Colombia, (UMELISA® Dengue IgM, Centro de Inmunoensayo Instituto Pedro Kouri, La Habana, Cuba).

\section{Evaluación clínica y morfológica}

A todos los recién nacidos se les realizó evaluación de crecimiento y desarrollo por pediatra, por medio de la Escala Abreviada de Desarrollo (EAD-1) del Ministerio de Salud de Colombia. Las áreas del desarrollo evaluadas fueron: motricidad gruesa, fino adaptativa, audición y lenguaje y personal social. Se realizó también evaluación física por médico genetista, según los parámetros establecidos en la historia genética del Departamento de Genética de la Facultad de Medicina de la Universidad de Antioquia.

\section{Recolección de la información y procesamiento de datos}

Los datos del recién nacido se obtuvieron de la historia clínica, los carnés prenatales y por interrogatorio a las madres. La recolección de los datos fue hecha por los investigadores.

\section{Análisis estadístico}

Los datos se procesaron en Epilnfo, versión 6.04c, y Microsoft Excel 97 (Microsoft Corp., Rendomd, WA). El seguimiento de los efectos en ambas cohortes se hizo en el primero, segundo y tercer trimestre del embarazo, en el momento del parto y a los 2 años de vida. A las variables cuantitativas se les calculó la media y la desviación estándar y a las variables cualitativas, las medidas de frecuencia absoluta y relativa. Las variables categóricas se compararon mediante la prueba exacta de Fisher. Las variables cuantitativas se compararon con la t de Student. Se consideró como nivel de significancia $p<0,05$.

\section{Resultados}

Al comparar el grupo de madres de las dos cohortes se encontró que eran semejantes en cuanto a la edad (t de Student, $p=0,95$ ), el número de embarazos (t de Student, $p=0,109$ ), y la vía del parto vaginal $\left(\chi^{2}, p=0,375\right)$ (cuadro 1$)$.

Dieciséis recién nacidos de la $C E$ fueron productos de madres clasificadas como casos confirmados de dengue, es decir, tuvieron anticuerpos IgM positivos para dengue y 6 recién nacidos fueron productos de madres clasificadas como casos compatibles. El 45,4\% (10/22) de los casos de dengue ocurrieron en el primer trimestre; el $36,4 \%$ (8/22) en el segundo trimestre y el 18,2\% (4/22) en el último trimestre (11).

En las historias clínicas revisadas y según el interrogatorio a las madres no se documentó dengue en ninguno de los recién nacidos. Tampoco se registraron muertes en los recién nacidos.

Cuadro 1. Características de las madres de los recién nacidos de la cohorte expuestas y no expuesta.

\begin{tabular}{|c|c|c|}
\hline Características & $\begin{array}{l}\text { Cohorte } \\
\text { expuesta }\end{array}$ & $\begin{array}{c}\text { Cohorte } \\
\text { no expuesta }\end{array}$ \\
\hline $\begin{array}{l}\text { Promedio de edad } \\
\text { de la madre* }\end{array}$ & 23.7 & 23.6 \\
\hline Promedio de embarazos ** & 2.2 & 1.9 \\
\hline Porcentaje de parto vaginal & 54.2 & 66.7 \\
\hline
\end{tabular}

* t de Student, $p=0,477 ;{ }^{* *} \mathrm{t}$ de Student, $p=0,109$;

${ }^{* \star *} \chi^{2}=0,78, p=0,375$ 


\section{Efectos sobre el feto y el recién nacido}

En el análisis de los efectos del dengue sobre el feto y el recién nacido se encontraron los siguientes hallazgos, los cuales se encuentran relacionados en el cuadro 2.

\section{Presencia de prematurez}

Se presentaron tres casos de nacimiento de recién nacidos con menos de 37 semanas de gestación $(13,6 \%, 3 / 22)$ en las pacientes de la CE, dos de los cuales fueron concomitantes con el dengue y otro posterior. En la CNE no se presentó ningún caso. No hubo diferencia estadísticamente significativa entre las cohortes de estudio (prueba de Fisher: $p=0,101$ ).

\section{Presencia de sufrimiento fetal}

Se presentaron tres niños de la CE con sufrimiento fetal en el momento del parto $(13,6 \%)$. Dos de estos eventos fueron concomitantes con el dengue y el otro al mes de la enfermedad. En el primer caso, al cuarto día del inicio de los síntomas del dengue en la madre, se desencadenó el parto prematuro (edad gestacional: 30 semanas) y simultáneamente hubo sufrimiento fetal agudo. La madre desarrolló oligoamnios severo e hipertensión inducida por el embarazo. El peso del recién nacido fue de $1.300 \mathrm{~g}$ y la talla de $40 \mathrm{~cm}$. El puntaje de Apgar a los 5 minutos fue de 7 y a los 10 minutos, 10.

En el segundo caso, el parto se desencadenó a los 4 días de la fecha del inicio de síntomas del dengue, a las 39 semanas de gestación. El peso del recién nacido al nacer fue de $3.400 \mathrm{~g}$ y la talla de $50 \mathrm{~cm}$. El puntaje de Apgar a los 5 minutos fue de 2 y a los 10 minutos, 10 .

En el tercer caso, el parto ocurrió a las 37 semanas de gestación, un mes después del dengue. El recién nacido presentó apnea respiratoria e hipotonía y hubo necesidad de practicar maniobras de reanimación en el momento de nacer, con buena respuesta. El peso al nacer fue $2.750 \mathrm{~g}$ y la talla de $48 \mathrm{~cm}$. El puntaje de Apgar a los 5 minutos fue de 4 y a los 10 minutos, 10 . En la CNE no se presentó ningún caso de sufrimiento fetal. No hubo diferencia estadísticamente significativa entre las cohortes de estudio (prueba de Fisher: $p=0,101)$.

\section{Presencia de retardo del crecimiento intrauterino}

Se presentó un recién nacido con retardo en el crecimiento intrauterino detectado desde el primer trimestre del embarazo en la cohorte expuesta.

Cuadro 2. Efectos del virus del dengue durante el embarazo, sobre el feto y el recién nacido. Medellín, Colombia, 1998.

\begin{tabular}{|c|c|c|c|c|c|}
\hline \multirow[t]{3}{*}{ Cohortes de estudio } & \multicolumn{2}{|c|}{$\begin{array}{l}\text { Efecto en el feto } \\
\text { y en el recién nacido }\end{array}$} & \multirow[t]{3}{*}{$\begin{array}{l}\text { Prueba de Fisher } \\
\text { Valor de } p\end{array}$} & \multirow[t]{3}{*}{$\begin{array}{l}\text { Concomitante } \\
\text { con dengue }\end{array}$} & \multirow[t]{3}{*}{$\begin{array}{l}\text { Posterior } \\
\text { al dengue }\end{array}$} \\
\hline & \multicolumn{2}{|c|}{ Prematurez } & & & \\
\hline & $<37$ sem & $\geq 37$ sem & & & \\
\hline Madre con dengue & 3 & 19 & 0,101 & 2 & 1 \\
\hline \multirow[t]{2}{*}{ Madre sin dengue } & 0 & 24 & & & \\
\hline & \multicolumn{2}{|c|}{ Sufrimiento fetal } & & & \\
\hline Madre con dengue & 3 & 19 & 0,101 & 2 & 1 \\
\hline \multirow[t]{3}{*}{ Madre sin dengue } & 0 & 24 & & & \\
\hline & \multicolumn{2}{|c|}{ Bajo peso al nacer } & & & \\
\hline & $<2500 \mathrm{~g}$ & $\geq 2500$ & & & \\
\hline Madre con dengue & 4 & 18 & 0,045 & - & - \\
\hline \multirow[t]{2}{*}{ Madre sin dengue } & 0 & 24 & & & \\
\hline & \multicolumn{2}{|c|}{ Defectos congénitos } & & & \\
\hline Madre con dengue & 3 & 19 & 0,101 & - & - \\
\hline \multirow[t]{2}{*}{ Madre sin dengue } & 0 & 24 & & & \\
\hline & \multicolumn{2}{|c|}{$\begin{array}{l}\text { Retardo del crecimiento } \\
\text { intrauterino }\end{array}$} & & & \\
\hline Madre con dengue & 1 & 21 & 0,47 & - & - \\
\hline Madre sin dengue & 0 & 24 & & & \\
\hline
\end{tabular}


Nació a las 40 semanas de gestación con un peso de $1.750 \mathrm{~g}$ y talla de $43 \mathrm{~cm}$. La madre sufrió dengue en el primer trimestre, pero también tenía el antecedente de hábito de fumar. En la CNE no se presentó ningún feto con retardo en el crecimiento intrauterino.

\section{Puntuación de Apgar}

Sólo se pudieron obtener datos sobre puntuación de Apgar a los 5 minutos en 8 de los 22 recién nacidos de la CE, de los cuales, 3 niños tuvieron puntajes inferiores o iguales a 7. El promedio de la puntuación de Apgar en este grupo fue de 7,4, con variaciones entre 2 y 10 . Entre los 24 recién nacidos de la CNE se obtuvieron datos de 15 niños; el puntaje promedio del Apgar fue de 9,9, con variaciones entre 9 y 10. La puntuación de Apgar a los 10 minutos se pudo obtener en 12 recién nacidos de la $C E$, con un valor promedio de 8,9 y en los recién nacidos de la CNE se obtuvo en 20 niños con un promedio de 9,9.

\section{Presencia de bajo peso al nacer}

En la CE se presentaron 4 casos de recién nacidos que pesaron menos de $2.500 \mathrm{~g}$ en el momento del nacimiento $(18,8 \%)$, con un valor promedio de $2.909,9 \mathrm{~g}$, con variaciones entre 1.200 y $4.000 \mathrm{~g}$. En la CNE, todos los recién nacidos tuvieron peso mayor de $2.500 \mathrm{~g}$, con un valor promedio de 3 205,7 y con variaciones entre $2.547 \mathrm{~g}$ y el máximo de $3.750 \mathrm{~g}$. Hubo diferencia estadísticamente significativa entre las cohortes de estudio (prueba exacta de Fisher: $p=0,045$ ).

\section{Presencia de defectos congénitos}

Se evaluaron por médico genetista los 22 niños de la cohorte expuesta y 24 de la cohorte no expuesta. Se detectaron 3 niños $(13,6 \%)$ de la CE con defectos congénitos de tipo malformación: 1) una niña con labio y paladar fisurados, foveolas auriculares y subpalpebral izquierda y polidactilia mínima; en este caso, la madre tuvo dengue en el segundo trimestre del embarazo; 2) un niño con displasia ectodérmica hipohidrótica, cuya madre presentó dengue en el tercer trimestre del embarazo; 3) un niño con polidactilia preaxial, la cual ya estaba corregida en el momento de la evaluación por el genetista; en este caso, la madre presentó dengue en el primer trimestre del embarazo. En la CNE no se presentaron casos de niños con defectos congénitos.

\section{Evaluación del crecimiento y desarrollo}

Se evaluaron por médico pediatra 21 niños de la cohorte expuesta y 24 de la cohorte no expuesta. La edad promedio en que fueron evaluados los recién nacidos fue de 22 meses, 20 meses en la CE y 22 meses en la CNE.

\section{Motricidad gruesa y fino adaptativa}

Se presentaron dos niños con desarrollo inadecuado de la motricidad gruesa y fino adaptativa en la CE (10,0\%). Todos los niños evaluados de la CNE presentaron un desarrollo adecuado de la motricidad gruesa y sólo uno presentó un desarrollo inadecuado del área fino adaptativa.

\section{Audición y lenguaje}

Se presentaron tres niños con desarrollo inadecuado del área de la audición y el lenguaje $(14,0 \%)$ en la CE. Ningún niño de la CNE presentó problemas en el área de la audición.

\section{Personal social}

Se presentaron 3 niños (14\%) con desarrollo inadecuado del área personal social en la CE. Uno de estos niños también tenía trastorno de la audición y el lenguaje. En la CNE se presentaron 2 niños (8\%) con desarrollo no adecuado del área personal social.

Al totalizar los puntajes obtenidos por cada uno de los niños en las diferentes áreas del desarrollo evaluadas mediante la EAD-1, sólo se presentó un niño (4\%) de la CE con desarrollo inadecuado y ninguno en la CNE. No hubo diferencia estadísticamente significativa entre los niños de ambas cohortes.

No ocurrió ninguna muerte fetal.

\section{Discusión}

En el presente trabajo se estudiaron 22 recién nacidos cuyas madres desarrollaron enfermedad por el virus del dengue durante el embarazo, en el transcurso de la epidemia ocurrida en 1998 en Medellín y se compararon con 24 recién nacidos de gestantes que no sufrieron dengue. 
Tres de los 22 recién nacidos (13,6\%) hijos de madres con dengue fueron prematuros; 2 eventos fueron concomitantes con dengue y uno posterior. Estos hallazgos coinciden con los informados por Carles y colaboradores (10), quienes en su estudio de 38 embarazadas con dengue de la Guyana Francesa, informaron un 22\% de prematurez como consecuencia del dengue. Así mismo, Poli y colaboradores (14) en un estudio de 5 embarazadas con dengue, describieron un caso de prematurez asociado con la infección. Sin embargo, Figueiredo y colaboradores (5) en su estudio de 10 casos de embarazadas con dengue en Brasil, relataron partos en edades gestacionales iguales o mayores de 38 semanas, es decir, no encontraron ningún caso de prematurez asociado con el dengue.

El $13,6 \%$ de los recién nacidos (3/22) de la CE presentó sufrimiento fetal en el momento del parto. Carles y colaboradores (10) describieron 4 casos de sufrimiento fetal durante el parto en su estudio de 38 gestantes con dengue. Otros autores en Cuba, como Fernández y colaboradores (6) en su estudio de 59 embarazadas con dengue, y Cantelar y Molina (3) en 35 gestantes con dengue, no informaron ningún caso de sufrimiento fetal relacionado con esta infección.

En el presente estudio, el índice de Apgar a los 5 minutos se obtuvo en $8 / 22$ recién nacidos de la $\mathrm{CE}, \mathrm{y}$ en tres de ellos se encontró el puntaje por debajo de 7. Cantelar y Molina (3) en Cuba informaron que a los 5 minutos de vida extrauterina el $100 \%$ de los recién nacidos presentó una puntuación entre 7 y 10 . Figueiredo y colaboradores (5) informaron índices de Apgar normales. Los resultados de la puntuación del Apgar a los 5 minutos fueron más bajos en la CE; sin embargo, las observaciones fueron muy pocas para dar resultados concluyentes.

El 18,8\% (4/22) de los recién nacidos de la CE tuvo un peso menor de $2.500 \mathrm{~g}$ en el momento del nacimiento. En la CNE, el $100 \%$ tuvo un peso mayor de $2.500 \mathrm{~g}$. Cantelar y Molina (3) encontraron que la proporción de recién nacidos con peso superior a los $2.500 \mathrm{~g}$ no mostró diferencias significativas entre los grupos que evaluaron. Figueiredo y colaboradores (5) informaron peso normal en los recién nacidos de las 10 gestantes con dengue estudiadas. Poli y colaboradores (14) describieron un recién nacido con peso inferior a los $2.500 \mathrm{~g}$, de los 5 estudiados. Chye y colaboradores (15) informaron un recién nacido de $2.200 \mathrm{~g}$ en uno de los dos casos que describieron.

La presentación de defectos congénitos de tipo malformativo, se presentó en el 13,6\% (3/22) de los recién nacidos de la CE, aparentemente no relacionados con dengue. Fernández y colaboradores (6) describieron que los niños de las madres con dengue durante el embarazo que fueron evaluados a los 5 años, mostraron un desarrollo psicomotor normal, descartando así un efecto teratogénico de detección tardía. Figueiredo y colaboradores (5) también informaron ausencia de malformación fetal. Carles y colaboradores (10), Poli y colaboradores (14) y Chye y colaboradores (15), tampoco detectaron defectos congénitos. Sin embargo, Sharma y Gulati (8) describieron un aumento de los casos de recién nacidos con defecto del tubo neural en una ciudad de la India. Este evento estuvo precedido de una epidemia de dengue en la que, al menos, un miembro de cada familia estuvo afectado y coincidió con el primer trimestre de embarazo de las gestantes. Los autores sugieren que este grupo de niños con defectos del tubo neural puede deberse a dengue en la gestante.

La evaluación del crecimiento y el desarrollo de los niños a los dos años de la infección materna de las dos cohortes, fue adecuada, lo cual coincide con los datos de Fernández y colaboradores en Cuba (6).

No se presentaron muertes fetales in utero en este estudio, hallazgo que difiere de los reportados por Carles y colaboradores (10) en su estudio de 38 pacientes, en el que hubo 5 óbitos fetales in utero.

Estos resultados preliminares muestran que los fetos y recién nacidos de mujeres que desarrollaron la enfermedad por dengue durante la gestación tuvieron mayor riesgo de bajo peso al nacer y presentaron con mayor frecuencia prematurez y sufrimiento fetal, pero se requiere continuar con esta línea de investigación para que las diferencias encontradas entre los grupos 
puedan ser confirmadas con un tamaño de muestra mayor. Sin embargo, ante los resultados del presente estudio, se debe alertar sobre la necesidad de vigilar en forma estrecha a las mujeres embarazadas con dengue, dados los efectos nocivos sobre la salud del recién nacido. Es importante destacar las discrepancias de los efectos del dengue durante el embarazo sobre el feto y en el recién nacido que informan los diferentes autores en la literatura. Una posible razón podría ser la diferencia en la severidad de las formas clínicas del dengue, determinadas por factores como la infección secuencial, la virulencia de las cepas, las características genéticas y los aspectos epidemiológicos (16-18), los cuales podrían influir en la presentación y la severidad de los efectos mórbidos sobre la gestación (11). En cuanto a los efectos de la fiebre sobre el embarazo, Nybo Andersen y colaboradores (19) en un estudio de cohorte con 24.000 mujeres, no encontraron evidencia de que la fiebre en las primeras 16 semanas de embarazo estuviera asociada con riesgo de muerte fetal y que la fiebre por sí misma no es factor de riesgo de muerte fetal. Simpson y colaboradores (20) sugieren también que la infección febril común es causa infrecuente de aborto. Sin embargo, Kline y colaboradores (20), en un estudio de casos y controles encontró asociación entre la fiebre y el aborto (odds ratio: 2,96 [IC95\%: 1,99-4,41]). Por lo tanto, la fiebre puede estar influenciando también en la aparición de efectos negativos en el embarazo y en el feto.

\section{Agradecimientos}

A la Dirección Seccional de Salud de Antioquia por la financiación del estudio; a Andrés Uribe, médico pediatra, por la evaluación de los recién nacidos y a Margarita Arboleda, médica epidemióloga del Instituto Colombiano de Medicina Tropical, por su colaboración en el análisis de la información.

\section{Referencias}

1. Instituto Nacional de Salud, Ministerio de Salud. Sivigila, Sistema de Vigilancia en Salud Pública 2002: 52:3-4.

2. Sierra ML, Vélez LM, Castañeda AM, Galeano LA, Molina, Tabares $\mathbf{Z}$ et al. Análisis de la morbimortalidad. Diagnóstico de la situación de salud en Antioquia. Revista Epidemiológica de Antioquia 2000;25:83-205.
3. Cantelar N, Molina LA. Dengue. Estudio clínico serológico en madres y recién nacidos. Trabajo preliminar. Rev Cub Med Trop 1981;33:96-105.

4. Chong KY, Lin KC. A preliminary report of the fetal effects of dengue infection in pregnancy. Kao Hsiung I Hsuch Ko Hsueh Tsa Chih Jan 1989;5:31-4.

5. Figueiredo LT, Carlucci RH, Duarte G. Estudo prospectivo com lactantes cujas maes tiveram dengue durante a gravidez. Rev Inst Med Trop Sao Paulo 1994;36:417-21.

6. Fernández $\mathbf{R}$, Rodríguez $\mathbf{T}$, Borbonet $\mathbf{F}$, Vásques S, Guzmán MG, Kouri G. Estudio de la relación dengue-embarazo en un grupo de madres cubanas. Rev Cub Med Trop 1994;46:46-8.

7. Bunyavejchevin $\mathbf{S}$, Tanawattanacharoen $\mathbf{S}$, Taechakraichana N, Thisyakorn U, Tannirandom T, Limpaphayom K. Dengue haemorrhagic fever during pregnancy: antepartum, intrapartum and postpartum management. J Obstet Gynaecol Res 1997;23:4458.

8. Sharma JB, Gulati N. Potential relationship between dengue fever and neural tube defects in northern district of India. Int J Gynaecol Obstet 1992;39:291-5.

9. Carles G, Peiffer H, Talarmin A. Effects of dengue fever during pregnancy in French Guiana. Clin Infect Dis 1999;28:637-40.

10. Carles G, Talarmin A, Peneau C, Bertsh M. Dengue fever and pregnancy. A study of 38 cases in French Guiana. J Gynecol Obstet Biol Reprod 2000;29:758-62.

11. Restrepo BN, Isaza DM, Salazar CL, Upegui G, Duque CL, Ospina $\mathbf{M}$ et al. Efectos del virus del dengue durante el embarazo, Medellín, Colombia. Infectio 2002;6:197-203.

12. Dirección Seccional de Salud de Antioquia. Protocolos de vigilancia epidemiológica. Guías integrales de atención. Quinta edición. Medellín: Ed. Dirección Seccional; 2001. p.142-8.

13. Júbiz A. Complicaciones obstétricas. En: Botero J, Júbiz A, Henao G, editores. Obstetricia y ginecología. Texto integrado. Sexta edición. Medellín: Quebecor Impreandes; 1999. p.209-26.

14. Poli L, Chungue E, Soulingnac $\mathbf{O}$, Gestas $P$, Kuo $P$, Papuin-Rauzi M. Materno-fetal dengue. Apropos of 5 casos observed during the epidemics in Tahiti (1989). Bull Soc Pathol Exot 1991;84:513-21.

15. Chye JK, Lim CT, Ng KB, Lim JMH, George R, Lam SK. Vertical transmission of dengue. Clin Infect Dis 1997; 25:1347-7.

16. Guzmán MG, Kouri G, Valdes L, Bravo J, Alvarez $\mathbf{M}$, Vasquez $\mathbf{S}$ et al. Epidemiologic studies of dengue in Santiago de Cuba, 1997. Am J Epidemiol 2000;152: 793-9. 
17. Guzmán MG, Kouri G, Bravo J, Soler M, Morier L, Vasques et al. Dengue en Cuba: historia de una epidemia. Rev Cub Med Trop 1988;40:29-49.

18. Bravo JR, Guzmán MG, Kouri G. Why dengue hemorrhagic fever in Cuba? 1. Individual risk factors for dengue hemorrhagic fever/dengue shock syndrome. Trans R Soc Trop Med Hyg 1987;81:816-20.

19. Nybo Andersen A-M, Vastrup Pernille, Wohlfahrt J, Kragh P. Fever in pregnancy and risk of fetal death: a cohort study. Lancet 2002;360:1552-6.
20. Simpson JL, Gray RH, Queenan JT, et al. Further evidence that infection is an infrequent cause of first trimester spontaneous abortion. Hum Reprod 1996;11: 2058-60.

21. Kline J. Stein Z, Susser M, Warburton D. Fever during pregnancy and spontaneous abortion. Am J Epidemiol 1985;121:832-42. 